\title{
LncRNA GASL1 inhibits growth and promotes expression of apoptosis-associated proteins in prostate carcinoma cells through GLUT-1
}

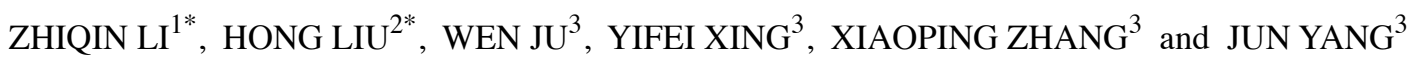 \\ ${ }^{1}$ Department of Pharmacy, Tongji Hospital, Tongji Medical College, Huazhong University of \\ Science and Technology, Wuhan, Hubei 430030; Departments of ${ }^{2}$ Critical Care Medicine, \\ Institute of Anesthesiology and Critical Care Medicine, and ${ }^{3}$ Urology, Union Hospital, \\ Tongji Medical College, Huazhong University of Science and Technology, Wuhan, Hubei 430022, P.R. China
}

Received May 15, 2018; Accepted January 7, 2019

DOI: $10.3892 / \mathrm{ol} .2019 .10244$

\begin{abstract}
Growth-arrest-associated long non-coding (lnc) RNA 1 (GASL1) is as newly identified lncRNA that is associated with liver cancer. The present study aimed to investigate the role of GASL1 in prostate carcinoma (PC). Expression levels of GASL1 in prostate tissues and sera from patients with PC and from healthy subjects were detected by reverse transcription-quantitative polymerase chain reaction. Receiver operating characteristic and survival curve analyses were performed to evaluate the diagnostic and prognostic values of GASL1 for patients with PC. A GASL1 expression vector was transfected into $\mathrm{PC}$ cells prior to assessment of cell proliferation and expression of B cell lymphoma 2 (Bcl-2) and glucose transporter 1 (GLUT-1) by Cell Counting Kit- 8 and western blotting, respectively. The results demonstrated that GASL1 was significantly downregulated in the tissue and serum of patients with PC compared to those of healthy subjects. In addition, GASL1 was used to distinguish patients with PC from healthy controls, and low expression levels of GASL1 were associated with short survival time. Expression levels of GASL1 were significantly associated with tumor size. GASL1 overexpression inhibited PC cell growth. Overexpression of GASL1 upregulated Bcl-2 expression and downregulated GLUT-1 expression. In conclusion, these data suggested that lncRNA GASL1 may inhibit PC cell proliferation by targeting GLUT-1.
\end{abstract}

Correspondence to: Professor Jun Yang, Department of Urology, Union Hospital, Tongji Medical College, Huazhong University of Science and Technology, 1277 Jiefang Avenue, Wuhan, Hubei 430022, P.R. China

E-mail: jizqag3@163.com

*Contributed equally

Key words: long non-coding RNA, growth-arrest-associated long non-coding RNA 1, prostate carcinoma, B cell lymphoma 2, glucose transporter 1

\section{Introduction}

Prostate carcinoma (PC) is one of the most frequently diagnosed malignancies worldwide and represents the second most common cause of cancer-associated mortality (1). Treatment outcomes of early stage PC are usually satisfactory (2); however, owing to the lack of symptoms of PC, many patients are diagnosed with existing tumor metastasis, which is a major cause of treatment failure and poor postsurgical survival (3). Currently, the incidence rate of PC is increasing, particularly in developing countries, including China, where PC is becoming a major public health problem (4). The pathogenesis of PC has not yet been entirely elucidated, and further investigation of the underlying molecular mechanism of PC development remains crucial to improving $\mathrm{PC}$ treatment.

B cell lymphoma 2 (Bcl-2) and glucose transporter 1 (GLUT-1) are involved in the pathogenesis of various types of malignancy, including PC $(5,6)$. Long non-coding (lnc) RNAs are a group of non-coding RNAs that possess crucial functions in normal and pathological physiological processes involved in numerous human diseases and malignancies, including different types of cancer (7). IncRNAs serve their roles by regulating the expression of downstream target genes that may be critical factors in human cancer (7). Therefore, the regulation of certain key lncRNAs may benefit the treatment of disease. Previous studies have reported that Bcl-2 and GLUT-1 interact with numerous IncRNAs involved in various biological processes $(8,9)$. Growth-arrest-associated lncRNA 1 (GASL1) is as newly identified lncRNA that acts as a tumor suppressor gene in liver cancer (10). In addition, GASL1 deletion enhances liver tumor growth, and low GASL1 expression levels are associated with poor survival of patients with liver cancer (11). The present study demonstrated that GASL1 inhibited cancer cell proliferation, upregulated BCL-2 and downregulated GLUT-1 expression levels in PC.

\section{Materials and methods}

Patients and specimens. The present study examined clinical data from 66 patients diagnosed with PC and treated 
at Tongji Hospital (Wuhan, China) between January 2011 and January 2013. Prostate biopsy tissues and serum samples were obtained from all patients. The inclusion criteria were as follows: i) Patients were diagnosed with PC by pathological examinations; ii) patients completed the whole treatment procedure at Tongji Hospital; iii) patients completed the follow-up and had complete clinical data record; and iv) patients received no treatment before admission. The exclusion criteria were as follows: i) At the time of the study, patients suffered or had suffered from other prostate diseases; ii) at the time of the study, patients suffered or had suffered from another cancer; iii) patients who were treated before admission; iv) patients who failed to complete the whole treatment procedure; and v) patients who died of other causes during the follow up. Prostate biopsy tissues and serum samples were also collected from 56 healthy subjects who received physiological examinations at Tongji Hospital between January 2011 and January 2013. These subjects underwent prostate biopsy to detect potential prostate lesions; prostate diseases were eventually excluded. The age of patients in the PC group ranged between 47 and 86 years (age, 68.9 \pm 5.1 years), and those in the healthy Control group ranged between 46 and 85 years (age, 69.1 \pm 5.0 years). No significant differences in age, smoking and drinking habits, and other basic clinical data, including body mass index and height, were identified between these two groups. The Ethics Committee of Tongji Medical College, Huazhong University of Science and Technology approved the present study. Written informed consents were obtained from all participants and/or their families.

Cell culture and transfection. The HPrEC human normal prostate epithelial cell line [cat. no. PCS-440-010 ${ }^{\mathrm{TM}}$; American Type Culture Collection (ATCC), Manassas, VA, USA] and the two human PC cell lines 22Rv1 (cat. no. CRL-2505 ${ }^{\mathrm{TM}}$; ATCC) and DU145 (cat. no. HTB-81 ${ }^{\mathrm{TM}}$; ATCC) were used in the present study. All cells were cultured with RPMI-1640 medium (Thermo Fisher Scientific, Inc., Waltham, MA, USA) with $10 \%$ fetal bovine serum (Thermo Fisher Scientific, Inc.) at $37^{\circ} \mathrm{C}$ with $5 \% \mathrm{CO}_{2}$.

An EcoRI-EcoRI fragment containing full length GASL1 cDNA was amplified by polymerase chain reaction (PCR), and a GASL1 expression vector was constructed by inserting this fragment into the pIRSE2-EGFP vector (Clontech Laboratories, Inc., Mountainview, CA, USA). GASL1 expression vector or empty pIRSE2-EGFP vector $(10 \mathrm{nM})$ was transfected into $6 \times 10^{5}$ cells using Lipofectamine ${ }^{\circledR} 2000$ reagent (cat. no. 11668-019; Invitrogen, Thermo Fisher Scientific, Inc.) following the manufacturer's protocol. Incubation with vectors was performed at $37^{\circ} \mathrm{C}$ for $6 \mathrm{~h}$. Subsequent experiments were performed $12 \mathrm{~h}$ after transfections. Expression of GASL1 was detected prior to and following subsequent experiments, and an overexpression rate $>150 \%(150-280 \%)$ was reached every time.

Cell counting Kit-8 (CCK-8) assay. Following transfection, HPrEC, 22Rv1 and DU145 cells were collected, washed with PBS and resuspended in RPMI-1640 medium (catalog no. 30-2001; ATCC) to the density of $3 \times 10^{4} / \mathrm{ml}$. Cell proliferation was measured by CCK- 8 assay. The highly water-soluble tetrazolium salt WST-8 is reduced by cellular dehydrogenases, which becomes orange in color (formazan); the amount of formazan is proportional to the number of living cells. Cells were cultured in a 96 well plate at a density of $3 \times 10^{3}$ cells $/ 100 \mu \mathrm{l} /$ well and placed in the incubator. CCK-8 solution (10 $\mu$; Sigma-Aldrich; Merck KGaA, Darmstadt, Germany) was added at 24, 48, 72 and $96 \mathrm{~h}$. Cells were then cultured for additional $4 \mathrm{~h}$ and $10 \mu \mathrm{l}$ DMSO was added. OD values were measured at $450 \mathrm{~nm}$ using Fisherbrand ${ }^{\mathrm{TM}}$ accuSkan $^{\mathrm{TM}}$ GO UV/Vis microplate spectrophotometer (Thermo Fisher Scientific, Inc.). Experiments were performed in triplicate. At 24, 48, 72 and $96 \mathrm{~h}$, cells were subjected to RNA extraction and reverse transcription-quantitative polymerase chain reaction (RT-qPCR) to confirm the overexpression of GASL1.

$R T$ - $q P C R$. TRIzol ${ }^{\circledR}$ reagent (Invitrogen; Thermo Fisher Scientific Inc.) was used to extract total RNA from HPrEC, 22Rv1 and DU145 cells ( $3 \times 10^{4}$ cells $\left./ \mathrm{ml}\right)$. To achieve complete cell lysis, tissues $(0.05 \mathrm{~g})$ were ground in liquid nitrogen prior to addition of TRIzol. RT was performed to synthesize cDNA using SuperScript IV Reverse Transcriptase kit (Thermo Fisher Scientific, Inc.) with the following conditions: $25^{\circ} \mathrm{C}$ for $6 \mathrm{~min}, 55^{\circ} \mathrm{C}$ for $20 \mathrm{~min}$ and $80^{\circ} \mathrm{C}$ for $10 \mathrm{~min}$. qPCR reaction systems were prepared using SYBR ${ }^{\circledR}$ Green Real-Time PCR Master Mixes (Thermo Fisher Scientific, Inc.). All primers were synthesized by Sangon Biotech Co., Ltd. (Shanghai, China). The PCR primers sequences were as follows: GASL1, forward 5'-CTGAGGCCAAAG TTTCCAAC-3', reverse 5'-CAGCCTGACTTTCCCTCT TCT-3'; and $\beta$-actin, forward 5'-GACCTCTATGCCAAC ACAGT-3', reverse 5'-AGTACTTGCGCTCAGGAGGA-3'. Thermocycling conditions were as follows: $95^{\circ} \mathrm{C}$ for $50 \mathrm{sec}$, followed by 40 cycles of $95^{\circ} \mathrm{C}$ for $15 \mathrm{sec}$ and $60^{\circ} \mathrm{C}$ for $35 \mathrm{sec}$. Expression of GASL1 was normalized to $\beta$-actin endogenous control using the $2^{-\Delta \Delta \mathrm{Cq}}$ method (12). This experiment was performed in triplicate.

Western blotting. Total protein was extracted from HPrEC, 22Rv1 and DU145 cell lines $12 \mathrm{~h}$ after transfection using RIPA Lysis and Extraction Buffer (Thermo Fisher Scientific Inc.) with a cell density of $3 \times 10^{4}$ cells $/ \mathrm{ml}$. The bicinchoninic acid assay was used to measure protein concentration. Proteins (30 $\mu \mathrm{g}$ per lane) were separated by $10 \%$ SDS-PAGE electrophoresis and transferred to a polyvinylidene fluoride membrane. Membranes were blocked with $5 \%$ skimmed milk at room temperature for $1 \mathrm{~h}$. Membranes were incubated with primary antibodies, including rabbit anti-Bcl-2 (cat. no. ab59348; 1:1,200; Abcam, Cambridge, UK), rabbit anti- Bcl-associated X (Bax; cat. no. ab32503; 1:1,200; Abcam, Cambridge, UK), rabbit anti-GLUT-1 (cat. no. ab15309; 1:1,200; Abcam) and rabbit anti-GAPDH antibody (cat. no. ab9485; 1:1,400; Abcam) at $4^{\circ} \mathrm{C}$ overnight. They were then incubated with horseradish peroxidase-conjugated goat anti-rabbit immunoglobulin G secondary antibody (cat. no. MBS435036; 1:1,000; MyBioSource, San Diego, CA, USA) for $2.5 \mathrm{~h}$ at room temperature. Enhanced Chemiluminescence Reagent (Sigma-Aldrich, Merck KGaA) was used to develop signal, which was detected using MYECL ${ }^{\mathrm{TM}}$ Imager (Thermo 

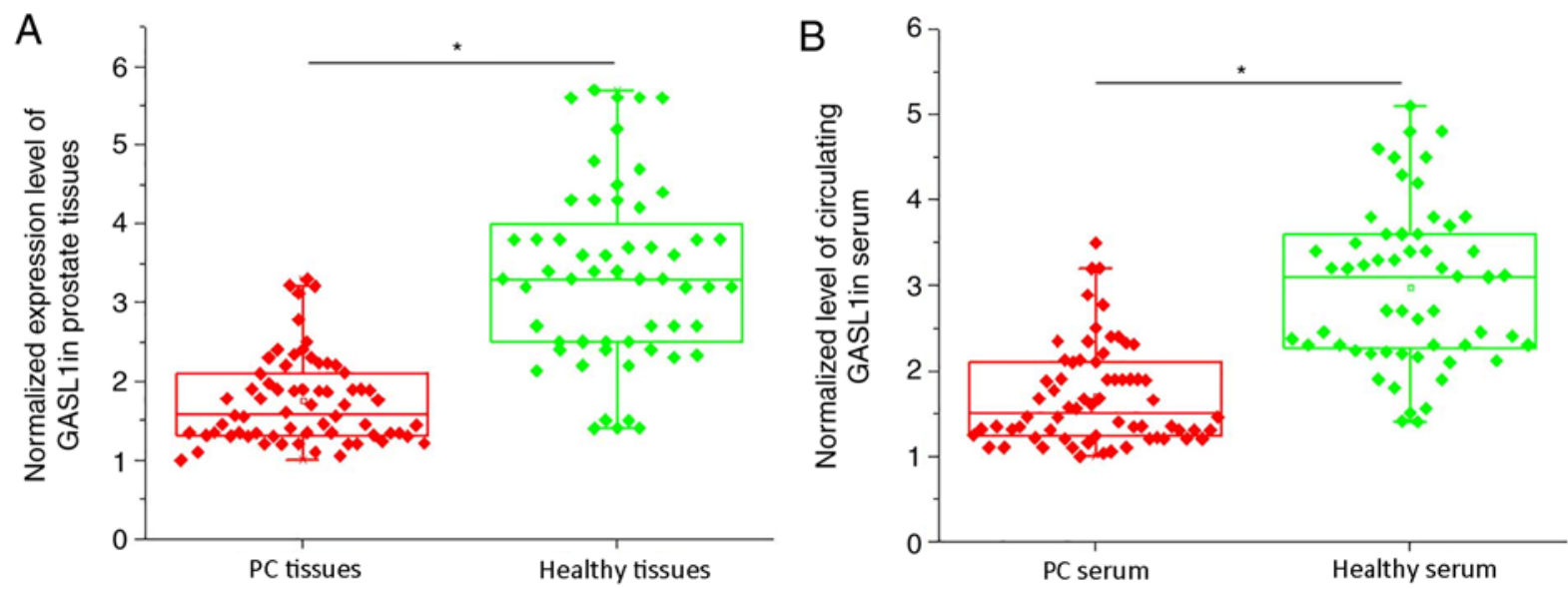

Figure 1. Expression levels of 1ncRNA GASL1 in prostate tissues and sera of patients with PC and healthy controls. (A and B) mRNA expression levels of lncRNA GASL1 in (A) prostate tissues and (B) sera in patients with PC and healthy controls were examined by reverse transcription-quantitative polymerase chain reaction. ${ }^{*} \mathrm{P}<0.05$. Each point represents the mean of three biological replicates. GASL1, growth-arrest-associated lncRNA 1. IncRNA, long non-coding RNA; PC, prostate cancer.
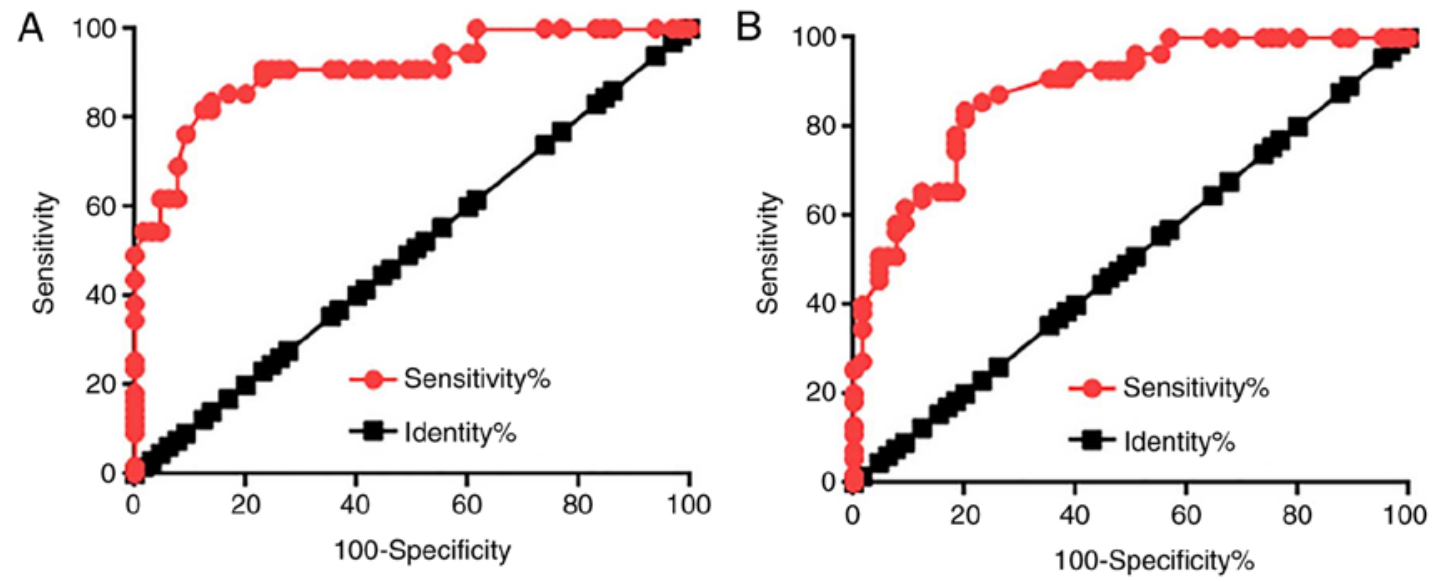

Figure 2. Diagnostic values of lncRNA GASL1 for PC. Receiver operating characteristic curve analysis of the diagnostic values of lncRNA GASL1 expression in (A) prostate tissues and (B) serum for PC. Red circles represent the diagnostic line and black squares represent the identity line. GASL1, growth-arrest-associated lncRNA 1; lncRNA, long non-coding RNA; PC, prostate cancer.

Fisher Scientific, Inc.). Expression levels of Bcl-2 and GLUT-1 were normalized to GAPDH endogenous control using Image J v1.6 software (National Institutes of Health, Bethesda, MD, USA). This experiment was performed in triplicate.

Statistical analysis. SPSS 19.0 software (IBM Corp., Armonk, NY, USA) was used to perform all statistical analysis. Expression levels of proteins, mRNA and cell proliferation data were expressed as the mean \pm standard deviation, and compared using unpaired Student's t-test (between 2 groups) or one-way analysis of variance followed by least significant difference test (among multiple groups). $\chi^{2}$ test was used to analyze the associations between GASL1 (in prostate tissues and serum) and patients' clinicopathological data. Receiver operating characteristic (ROC) curve was performed using patients with $\mathrm{PC}$ as true positive subjects and healthy controls as true negative subjects, and all parameters were default [95\% confidence interval (CI)]. Patients were divided into high and low expression group according to the median serum levels of GASL1

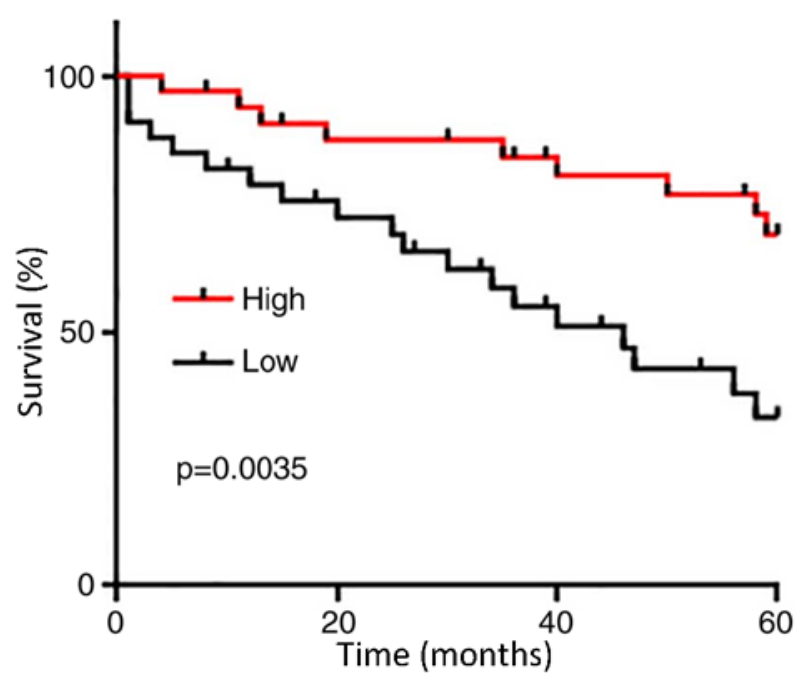

Figure 3. Prognostic values of serum lncRNA GASL1 for prostate cancer. Overall survival of patients with low serum level of GASL1 was significantly poorer compared with those with high serum level of GASL1. GASL1, growth-arrest-associated lncRNA 1; lncRNA, long non-coding RNA. 
Table I. Expression levels of growth-arrest-associated lncRNA 1 in prostate tissues were not associated with patients age, lifestyle habits including drinking and smoking and tumor distant metastasis.

\begin{tabular}{|c|c|c|c|c|c|}
\hline Characteristic & Cases & High-expression & Low-expression & $\chi^{2}$ & P-value \\
\hline \multicolumn{6}{|l|}{ Age (years) } \\
\hline$>70$ & 31 & 14 & 17 & \multirow[t]{2}{*}{0.55} & \multirow[t]{2}{*}{0.46} \\
\hline$\leq 70$ & 35 & 19 & 16 & & \\
\hline \multicolumn{6}{|l|}{ Drinking } \\
\hline Yes & 50 & 23 & 27 & \multirow[t]{2}{*}{1.32} & \multirow[t]{2}{*}{0.25} \\
\hline No & 16 & 10 & 6 & & \\
\hline \multicolumn{6}{|l|}{ Smoking } \\
\hline Yes & 44 & 20 & 24 & \multirow[t]{2}{*}{2.58} & \multirow[t]{2}{*}{0.11} \\
\hline No & 22 & 13 & 9 & & \\
\hline \multicolumn{6}{|c|}{ Primary tumor diameter $(\mathrm{cm})$} \\
\hline$>5$ & 30 & 10 & 20 & \multirow[t]{2}{*}{6.11} & \multirow[t]{2}{*}{0.01} \\
\hline$\leq 5$ & 36 & 23 & 13 & & \\
\hline \multicolumn{6}{|c|}{ Tumor distant metastasis } \\
\hline Yes & 23 & 13 & 10 & \multirow[t]{2}{*}{0.6} & \multirow[t]{2}{*}{0.44} \\
\hline No & 43 & 20 & 23 & & \\
\hline
\end{tabular}

Values in bold highlight significant P-values.

(1.64). Kaplan-Meier (KM) analysis was performed to plot survival curves, which were compared by log-rank test. $\mathrm{P}<0.05$ was considered to indicate a statistically significant difference.

\section{Results}

Comparison of expression levels of lncRNA GASL1 in prostate tissues and sera between patients with PC and healthy controls. mRNA expression levels of lncRNA GASL1 in prostate tissues and sera of patients with PC and healthy controls were detected by RT-qPCR. As shown in Fig. 1A, the expression levels of lncRNA GASL1 in PC tissues were significantly lower compared with healthy control tissues $(\mathrm{P}<0.05)$. In addition, the expression levels of circulating lncRNA GASL1 in serum were also significantly lower in PC tissues compared with healthy controls $(\mathrm{P}<0.05$; Fig. 1B). These data suggested that downregulation of IncRNA GASL1 may be involved in pathogenesis of PC.

Diagnostic values of lncRNA GASL1 for PC. Diagnostic values of lncRNA GASL1 expression in prostate tissues and sera for patients with PC were evaluated using ROC curve analysis. As demonstrated in Fig. 2A, the area under the curve (AUC) for GASL1 expression in prostate tissues used for PC diagnosis was 0.9076 with a standard error of 0.02718 and 95\% CI of 0.8543-0.9608. In addition, the AUC of GASL1 expression in serum used for PC diagnosis was 0.8811 with a SE of 0.02976 and $95 \%$ CI of 0.8228-0.9359 (Fig. 2B). These results suggested that lncRNA GASL1 may serve as a potential biomarker for PC.

Prognostic values of serum level of IncRNA GASL1 for PC. The 66 patients with $\mathrm{PC}$ were divided into high $(\mathrm{n}=33)$ and low $(n=33)$ expression groups according to the median serum level of GASL1 (1.64). KM was used to plot survival curves, which were compared using log rank test. As presented in Fig. 3, the overall survival of patients with low serum level of GASL1 was significantly poorer compared with patients with high serum level of GASL1 $(\mathrm{P}=0.0035)$. These data suggested that serum levels of GASL1 may serve as a potential prognostic biomarker for PC.

Association of IncRNA GASL1 expression levels with clinicopathological data of patients with PC. Associations between expression levels of IncRNA GASL1 in prostate tissues and sera from patients with PC clinicopathological data were analyzed by $\chi^{2}$ test. As presented in Tables I and II, expression levels of lncRNA GASL1 in prostate tissues and sera were not associated with patients age, lifestyle habits (including drinking and smoking) and existing of tumor distant metastasis. By contrast, expression levels of 1ncRNA GASL1 in prostate tissues and sera were associated with tumor size.

Effects of GASL1 overexpression on cell proliferation. Significantly lower expression levels of GASL1 were observed in 22Rv1 and DU145 cell lines compared with the HPrEC cell line $(\mathrm{P}<0.05$; Fig. 4A). According to the data in Tables I and II, GASL1 could hypothetically be involved in PC tumor growth. The effects of GASL1 overexpression on cell proliferation were assessed by CCK-8 assay. As demonstrated in Fig. 4B, GASL1 overexpression significantly inhibited 22Rv1 and DU145 cell proliferation compared with the Control groups at 96 h; however, GASL1 overexpression exhibited no observable effect on HPrEC cell proliferation. In addition, compared with the control and negative control groups, significant GASL1 overexpression was observed at different time points, which 
Table II. Expression levels of growth-arrest-associated lncRNA 1 in serum were significantly associated with tumor size.

\begin{tabular}{|c|c|c|c|c|c|}
\hline Characteristic & Cases & High-expression & Low-expression & $\chi^{2}$ & P-value \\
\hline \multicolumn{6}{|l|}{ Age (years) } \\
\hline$>70$ & 31 & 15 & 16 & \multirow[t]{2}{*}{0.1} & \multirow[t]{2}{*}{0.81} \\
\hline$\leq 70$ & 35 & 18 & 17 & & \\
\hline \multicolumn{6}{|l|}{ Drinking } \\
\hline Yes & 50 & 22 & 28 & \multirow[t]{2}{*}{3} & \multirow[t]{2}{*}{0.08} \\
\hline No & 16 & 11 & 5 & & \\
\hline \multicolumn{6}{|l|}{ Smoking } \\
\hline Yes & 44 & 19 & 25 & \multirow[t]{2}{*}{2.5} & \multirow[t]{2}{*}{0.12} \\
\hline No & 22 & 14 & 8 & & \\
\hline \multicolumn{6}{|c|}{ Primary tumor diameter $(\mathrm{cm})$} \\
\hline$>5$ & 30 & 11 & 19 & \multirow[t]{2}{*}{3.9} & \multirow[t]{2}{*}{0.047} \\
\hline$\leq 5$ & 36 & 22 & 14 & & \\
\hline \multicolumn{6}{|c|}{ Tumor distant metastasis } \\
\hline Yes & 23 & 13 & 10 & \multirow[t]{2}{*}{0.6} & \multirow[t]{2}{*}{0.44} \\
\hline No & 43 & 20 & 23 & & \\
\hline
\end{tabular}

Values in bold highlight significant P-values.

A

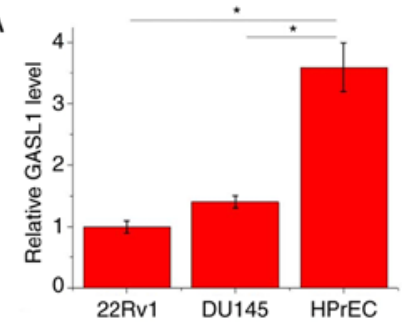

C

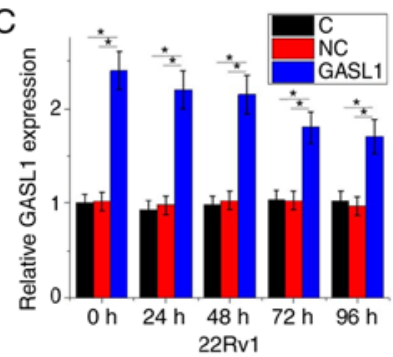

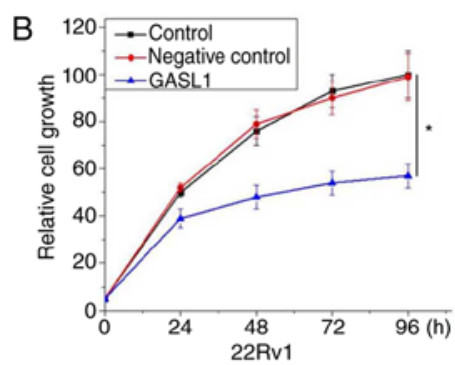
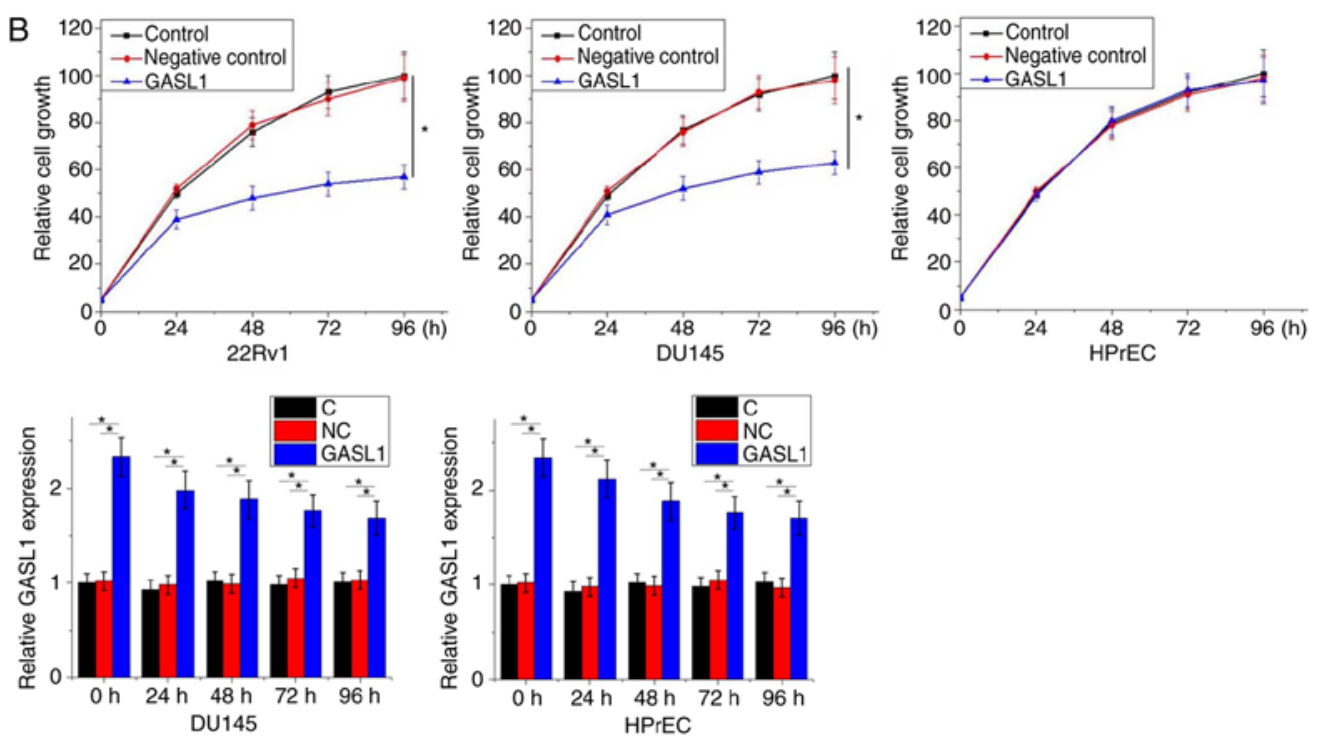

Figure 4. Effects of lncRNA GASL1 overexpression on cell proliferation. (A) Expression levels of GASL1 were significantly lower in the 22 Rv1 and DU145 cell lines compared with expression in the HPrEC cell line; P<0.05. (B) GASL1 overexpression significantly inhibited 22Rv1 and DU145 cell proliferation, but had no significant effect on HPrEC cell proliferation; "P $<0.05$ vs. control. (C) Overexpression of GASL1 was observed at different time points during CCK-8 assay; "P $<0.05$. Each data represent the mean of three biological replicates. C, control; GASL1, growth-arrest-associated lncRNA 1; lncRNA, long non-coding RNA; NC, negative control.

supports the effects of GASL1 overexpression on cancer cell proliferation $(\mathrm{P}<0.05$; Fig. $4 \mathrm{C})$.

Effects of GASL1 overexpression on Bcl-2, GLUT-1 and Bax expression. Bcl-2, GLUT-1 and Bax are associated with development of various types of malignancy $(8,9)$. In the present study, GASL1 overexpression (12 $\mathrm{h}$ following transfection) significantly increased $\mathrm{Bcl}-2$ expression and significantly inhibited GLUT-1 expression in 22Rv1 and DU145 cells
(Fig. 5A and B, respectively). However, GASL1 overexpression had no effect on Bcl-2 and GLUT-1 expression in the HPrEC cell line (Fig. 5C). GASL1 overexpression had no effect Bax expression in any of the three cell lines (Fig. 5A-C).

\section{Discussion}

GASL1 is a novel lncRNA characterized only in liver cancer (10). The present study demonstrated that GASL1 may 

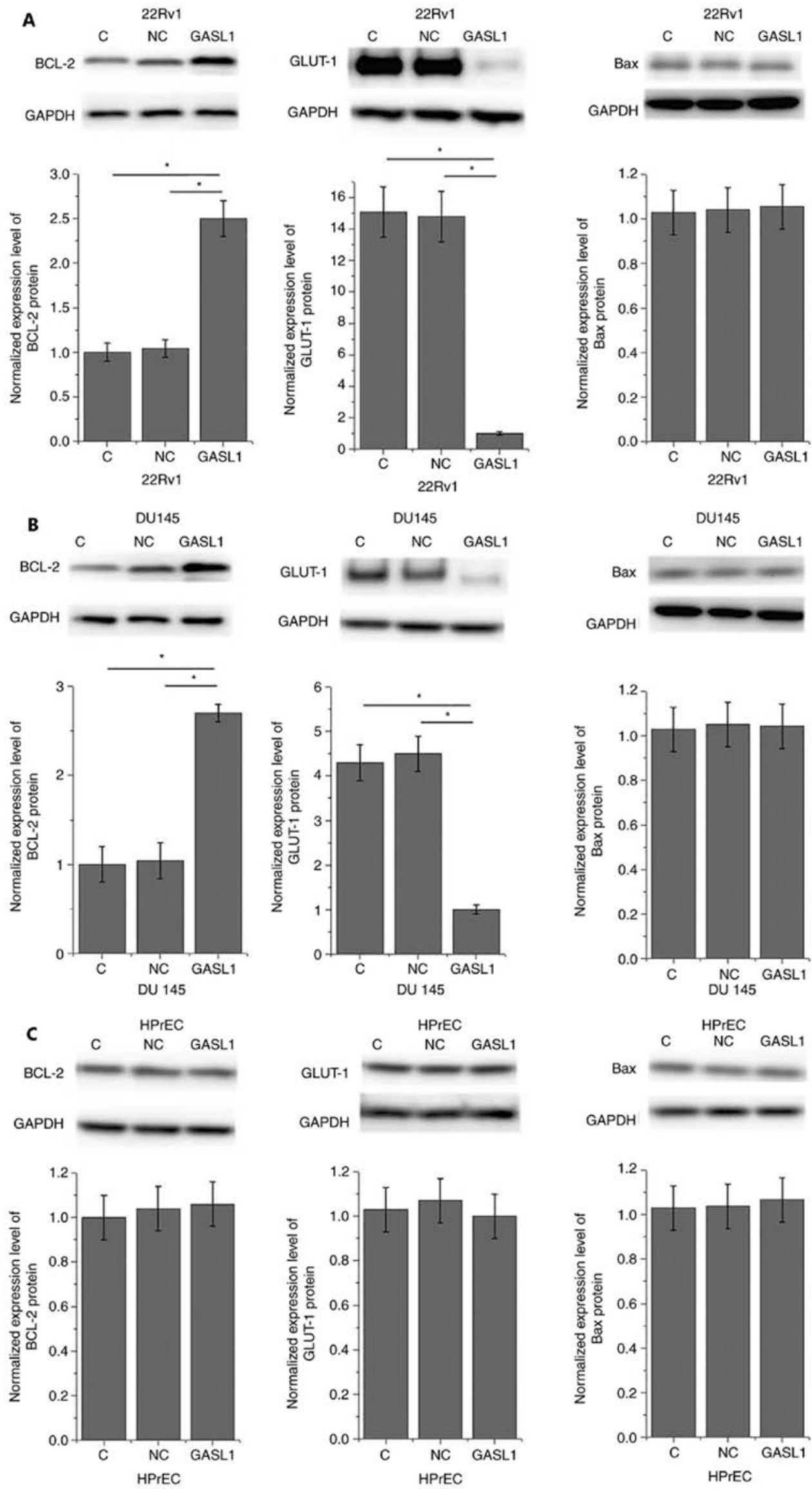

Figure 5. Effects of lncRNA GASL1 overexpression on Bcl-2, GLUT-1 and Bax expression. (A-C) GASL1 overexpression (12 h post-transfection) significantly increased Bcl-2 expression, but inhibited GLUT-1 expression in (A) 22Rv1 and (B) DU145 cells, but not in the (C) HPrEC cell line. GASL1 overexpression did not significantly affect Bax expression in the three cell lines. Each data represent the mean of three biological replicates; "P<0.05. C, control; Bax, Bcl-2-associated X; Bcl-2, B cell lymphoma 2; GASL1, growth-arrest-associated lncRNA 1; GLUT-1, glucose transporter 1; IncRNA, long non-coding RNA; $\mathrm{NC}$, negative control. 
serve a role as a tumor suppressor gene in PC by inhibiting cancer cell proliferation. The underlying mechanism of GASL1 in PC may be through GLUT-1 downregulation.

Genetic factors serve a central role in the pathogenesis of PC. Genetic polymorphism of glutathione S-transferase M1 and glutathione $\mathrm{S}$-transferase $\theta 1$ genes is closely associated with PC risk (11). A previous study reported that lncRNA polymorphisms may affect the occurrence of PC (12). In addition, numerous lncRNAs exhibit different expression patterns during PC progression and serve various roles as tumor suppressor genes or oncogenes (13). GASL1 is downregulated in liver cancer, indicating that it may be a tumor suppressor gene (10). In the present study, expression levels of lncRNA GASL1 were significantly lower in patients with PC compared with healthy controls in prostate tissues and in sera, which indicated that GASL1 may serve a role as a tumor suppressor gene in PC.

Blood biomarkers are crucial for the diagnosis of numerous human diseases (14). In the present study, GASL1 was differentially expressed in patients with PC and healthy controls, and ROC curve analysis revealed that expression levels of lncRNA GASL1 in prostate tissues and sera may be used to distinguish patients with PC from healthy controls. The results also revealed that low expression level of GASL1 was closely associated with poor postoperative survival. Furthermore, expression levels of lncRNA GASL1 in prostate tissues and sera were not associated with patient age or lifestyle habits, which are factors known to affect the expression of certain IncRNAs (15-17). These results indicated that GASL1 may serve as a potential reliable and effective diagnostic and prognostic biomarker for PC. Compared with prostate biopsy, serum collection from patients is a less invasive procedure that can be performed in some cases when biopsy is not applicable. Notably, lncRNA GASL1 has not been characterized in other diseases. Multiple biomarkers may thus be combined to improve the diagnosis and prognosis.

In the present study, GASL1 expression was significantly associated with tumor size but not distant tumor metastasis, which suggested that GASL1 may be associated with tumor growth but not with metastasis in PC. Furthermore, CCK-8 assays revealed that GASL1 may be an inhibitor of cancer cell proliferation in PC. Bcl-2 is a member of the Bcl-2 protein family that regulates cell proliferation by inhibiting pro-apoptotic pathways or inducing anti-apoptotic pathways (18). GLUT-1 serves a major role in glucose metabolism and is usually upregulated in tumor tissues to promote tumor growth (19). In the present study, GASL1 overexpression increased Bcl-2 expression but inhibited GLUT-1 expression in PC cells. In addition, Bax is an anti-apoptotic protein (18). In the present study, GASL1 overexpression had no effect on Bax expression in PC and normal cell lines. GASL1 may therefore interact with multiple pathways and have various roles; however, the underlying mechanisms of action of GASL1 remain unclear. GASL1 may inhibit PC by downregulating GLUT-1. The stimulating role of GASL1 on Bcl-2 may reveal the complexity of the regulatory role of GASL1 on cell proliferation and apoptosis. In addition, previous studies reported that GASL1 regulates cell cycle and apoptosis (10). Therefore, the inhibition of cell proliferation observed after GASL1 expression may be due to apoptosis stimulation or cell cycle progression inhibition.
GASL1 overexpression had no effect on growth or Bcl-2 and GLUT-1 protein expressions in the HPrEC normal prostate epithelial cell line, which suggested that GASL1 may serve as a potential therapeutic target for treatment of PC. The present study had some limitations. Cell apoptosis and cell cycle were not examined, although they may have revealed functions of GASL1 on other aspects of PC; these analyses will be performed in a future study. However, results from the present study may provide guidance for future studies investigating the functionality of GASL1 in PC and other malignancies.

In conclusion, GASL1 was significantly downregulated in patients with PC compared with healthy controls, which suggested that GASL1 may serve as a potential diagnostic and prognostic biomarker in PC. In addition, expression levels of GASL1 were significantly associated with tumor size. GASL1 overexpression inhibited PC cell proliferation, upregulated Bcl-2 expression and downregulated GLUT-1 expression. Taken together, these data suggested that lncRNA GASL1 may inhibit PC cell proliferation by targeting GLUT-1.

\section{Acknowledgements}

Not applicable.

\section{Funding}

The study was supported by The National Natural Science Foundation (grant no. 30872924).

\section{Availability of data and materials}

All the data generated or analyzed during the current study are available from the corresponding author on reasonable request.

\section{Authors' contributions}

ZL and HL performed experiments. ZL, HL, WJ, YX, XZ and JY assisted with the experiments and statistical analysis. ZL, HL and JY wrote the manuscript. JY revised the manuscript. All authors read and approved the final manuscript.

\section{Ethics approval and consent to participate}

The Ethics Committee of Tongji Hospital, Tongji Medical College, Huazhong University of Science and Technology (Wuhan, China) approved the protocol. Written informed consent was provided by patients and healthy people involved.

\section{Patient consent for publication}

Not applicable.

\section{Competing interests}

The authors declare that they have no competing interests.

\section{References}

1. Siegel RL, Miller KD and Jemal A: Cancer statistics, 2018. CA Cancer J Clin 68: 7-30, 2018. 
2. Lepor H: Surgical treatment of prostate carcinoma. J Urol 197 (2S): S41-S42, 2017

3. Gundem G, Van Loo P, Kremeyer B, Alexandrov LB, Tubio JMC, Papaemmanuil E, Brewer DS, Kallio HML, Högnäs G, Annala M, et al: The evolutionary history of lethal metastatic prostate cancer. Nature 520: 353-357, 2015.

4. Yan W, Li H, Zhou Y, Huang Z, Rong S, Xia M, Ji Z, Chen J and Jiang Y: Prostate carcinoma spatial distribution patterns in Chinese men investigated with systematic transperineal ultrasound guided 11-region biopsy. Urol Oncol 27: 520-524, 2009.

5. Lian J, Wu X, He F, Karnak D, Tang W, Meng Y, Xiang D, Ji M, Lawrence TS and Xu L: A natural BH3 mimetic induces autophagy in apoptosis-resistant prostate cancer via modulating Bcl-2-Beclin1 interaction at endoplasmic reticulum. Cell Death Differ 18: 60-71, 2011.

6. Effert P, Beniers AJ, Tamimi Y, Handt S and Jakse G: Expression of glucose transporter 1 (Glut-1) in cell lines and clinical specimens from human prostate adenocarcinoma. Anticancer Res 24 3057-3063, 2004

7. Gutschner T and Diederichs S: The hallmarks of cancer: A long non-coding RNA point of view. RNA Biol 9: 703-719, 2012.

8. Schmidt LH, Görlich D, Spieker T, Rohde C, Schuler M, Mohr M, Humberg J, Sauer T, Thoenissen NH, Huge A, et al: Prognostic impact of Bcl-2 depends on tumor histology and expression of MALAT-1 lncRNA in non-small-cell lung cancer. J Thorac Oncol 9: 1294-1304, 2014.

9. Liu X and Gan B: lncRNA NBR2 modulates cancer cell sensitivity to phenformin through GLUT1. Cell Cycle 15: 3471-3481, 2016.

10. Gasri-Plotnitsky L, Ovadia A, Shamalov K, Nizri-Megnaji T, Meir S, Zurer I, Cohen CJ and Ginsberg D: A novel lncRNA, GASL1, inhibits cell proliferation and restricts E2F1 activity. Oncotarget 8: 23775-23786, 2017.

11. Malik SS, Kazmi Z, Fatima I, Shabbir R, Perveen S and Masood N: Genetic polymorphism of GSTM1 and GSTT1 and risk of prostatic carcinoma-a meta-analysis of 7,281 prostate cancer cases and 9,082 healthy controls. Asian Pac J Cancer Prev 17: 2629-2635, 2016.
12. Jin G, Sun J, Isaacs SD, Wiley KE, Kim ST, Chu LW, Zhang Z, Zhao H, Zheng SL, Isaacs WB and Xu J: Human polymorphisms at long non-coding RNAs (lncRNAs) and association with prostate cancer risk. Carcinogenesis 32: 1655-1659, 2011.

13. Zhou W, Wang Z, Tao Z, Ji L, Zhou B, Shen M and Tu H: Differential expression of long non-coding RNAs in prostatic carcinoma cell line $\mathrm{LNCaP}$ after occurrence of androgen independent transformation. Int J Clin Exp Pathol 10: 6665-6673, 2017.

14. Hori SS and Gambhir SS: Mathematical model identifies blood biomarker-based early cancer detection strategies and limitations. Sci Transl Med 3: 109ra116, 2011.

15. Grammatikakis I, Panda AC, Abdelmohsen K and Gorospe M: Long noncoding RNAs(lncRNAs) and the molecular hallmarks of aging. Aging (Albany NY) 6: 992-1009, 2014.

16. Mayfield RD: Emerging roles for ncRNAs in alcohol use disorders. Alcohol 60: 31-39, 2017

17. Wang J, Qiu M, Xu Y, Li M, Dong G, Mao Q, Yin R and Xu L: Long noncoding RNA CCAT2 correlates with smoking in esophageal squamous cell carcinoma. Tumour Biol 36: 5523-5528, 2015.

18. Li H, Li X, Bai M, Suo Y, Zhang G and Cao X: Matrine inhibited proliferation and increased apoptosis in human breast cancer MCF-7 cells via upregulation of Bax and downregulation of Bcl-2. Int J Clin Exp Pathol 8: 14793-14799, 2015.

19. Kurahara H, Maemura K, Mataki Y, Sakoda M, Iino S, Kawasaki Y, Arigami T, Mori S, Kijima Y, Ueno S, et al: Significance of glucose transporter type 1 (GLUT-1) expression in the therapeutic strategy for pancreatic ductal adenocarcinoma. Ann Surg Oncol 25: 1432-1439, 2018. 